\title{
Michael Epstein
}

\section{That thing is so annoying}

\section{How proactive chat helps us reach more users}

$\mathbf{T}^{\mathrm{t}}$

he comment came from a fellow librarian

when I asked if she had noticed our new proactive chat widget. Yes, she said, "that thing is so annoying." When I mentioned the percentage our chat requests were up by her eyes got wide and she replied, "Really?" Yes, really, and I was somewhat surprised myself. I should explain.

In April 2017, I along with two other reference librarians at my library were tasked with investigating reference trends

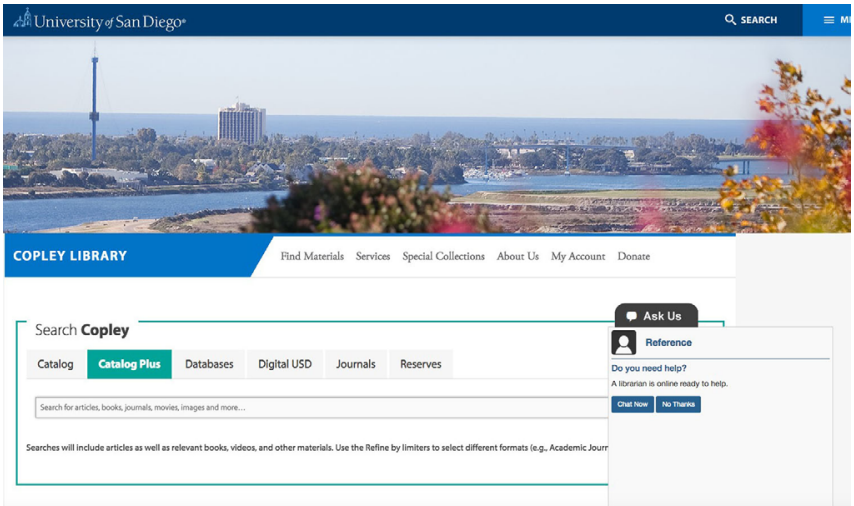

Figure 1. The chat widget on the Copy Library website. View this article online for a more detailed image.
LibAnswers version one system and did not offer proactive response. Fortunately, we had already planned to move to version two of LibAnswers in July 2017, and the new version included the ability to make the widget proactive. This meant that rather than just sitting at the bottom of the page, the widget would now slide up on its own to alert users that a librarian was available to help (see figure 1). The appearance of the widget tab remained the same and nothing was in academic libraries, as well as making recommendations on ways to better reach our users. Chat had been a particularly low point of contact for our reference transactions (5\% in fall 2016), and during our research we reviewed a few studies that found that adding a proactive element to a chat widget significantly increased chat transactions. ${ }^{1}$

We already had a chat widget that rested at the bottom of the screen on all our library website pages. When a user clicked on the widget's "Ask Us" tab, it would slide up the chat box. The widget was part of our done to alert users to the implementation of proactive chat.

Shortly after launch, I began to see an increase in chat requests. I was cautiously optimistic, since summer is generally a slow time for reference. I was curious to see what would happen when students returned for fall semester.

Michael Epstein is head of reference at the University of San Diego Copley Library, email: epsteinsandiego.edu

๑) 2018 Michael Epstein 
The proactive chat widget is set for a 60- second delay, hence the comment about it being annoying. While users can easily prevent the widget from sliding up again by clicking the "No Thanks" option, the widget resets if they move to a new page. It didn't take long to realize that the widget was having an impact. Reference librarians noticed a significant increase in chat requests during their reference desk shifts. Looking at the actual statistics confirmed that chat requests were up significantly compared to the previous year. A comparison of chats answered September 2017 through April 2018 with the same period for 2016 through 2017 revealed a nearly 600\% increase. This large statistical increase in chat requests led me to conclude that the previous widget was not being noticed by many users.

While we have not experimented with a shorter delay period for the proactive popup, I believe that giving users a little time to explore the site before prompting is more effective than an immediate prompt for help. One useful aspect of the system is the ability to see the web page the user was on when they initiated the chat. This gives us a clue about how they were navigating before initiating chat and where they may have gone astray.

For example, I have noticed that users sometimes initiate chats from pages that are either unrelated to the information they are seeking or just a bit off target. It's probable that patrons tried searching the site first and then initiated the chat when they got the popup. Prior to proactive chat, it is quite possible that these users would not have contacted us at all, due to not noticing that chat was an available service. Thus they may have spent more time on their own trying to find the proper sources or services. In this scenario, proactive chat can intervene to save users time and potential frustration in finding the information they need.

Analysis of chat transactions since launching the proactive widget indicates that more than $70 \%$ were recorded as research assistance type questions. This has led me to consider implementing proactive chat in other point of need interfaces, such as our discovery platform, databases, and online courseware systems. One consideration here is handling the potential additional volume of chats. During busy periods we now sometimes handle two or more chats at the same time.

Besides simply being more noticeable, I also think there may be a psychological component to proactive chat. We, in a sense, are directly contacting the user to invite them to ask us a question rather than them initiating first contact. This, I think, sends a message that we are not just available if needed, but are eager to offer assistance.

While proactive chat helps us to better connect with all our users, I believe that the increased visibility of our chat service is especially important for students in onlineonly programs and busy graduate students who are often off campus. Chat offers them an easy way to connect with a reference librarian and helps them understand that they do not have to visit the library to get assistance with their research.

One key takeaway from this experience has been that you don't necessarily have to make big changes to achieve large results. Something as simple as changing the behavior of a chat widget can turn out to be highly significant.

So, while proactive chat is no doubt sometimes annoying, there is also little doubt that it is an effective way of reaching users that need research assistance. I like to think of it as a friendly reminder that we are here and ready to help.

\section{Note}

1. Jan H. Kemp, Carolyn L. Ellis, and Krisellen Maloney, "Standing By to Help: Transforming Online Reference with a Proactive Chat System," The Journal of Academic Librarianship 41, no. 6 (2015): 764-770, doi:10.1016/j.acalib.2015.08.018; Jie Zhang and Nevin Mayer, "Proactive Chat Reference: Getting in the Users' Space," CERL News 75 (2014): 202-205. « 